\title{
Valeurs de référence de l'unité de dose collective professionnelle pour la mise en œuvre de la politique "ALARA" dans les centrales nucléaires d'Electricité de France
}

A. BERTHET*, P. BOUSSARD**, J. LOCHARD***, A. BRISSAUD*, Ph. ROLLIN ${ }^{\star \star \star \star}$, C. LEFAURE ${ }^{\star \star \star}$

RÉSUMÉ La mise en cuvre de la politique "ALARA" au niveau de la conception, l'exploitation et la maintenance des REP du parc EDF a pour but de maîtriser les expositions individuelle et collective et d'en assurer une gestion rationnelle. L'objectif de cette politique est, conformément aux recommandations de la CIPR 60, de faire en sorte que les expositions soient maintenues aussi bas que raisonnablement possible (nombre de personnes exposées et niveau d'exposition), et réparties de façon équitable tout en tenant compte des facteurs économiques et sociaux. Ceci implique qu'EDF se dote, entre autres outils, d'un système de valeurs monétaires de référence de l'unité de dose collective évitée. Après avoir évoqué les problèmes d'éthique associés et le consensus des experts internationaux en matière de relation dose-effet, l'article présente le modèle adopté, basé sur l'aversion à la dispersion des risques. II se traduit par une valeur de l'unité de dose collective qui augmente fortement quand les doses individuelles annuelles croissent et s'approchent des limites. Ce modèle est un outil d'aide à la décision qui permet de comparer rationnellement différentes solutions en vue d'optimiser les expositions professionnelles.

ABSTRACT Implementing the ALARA principle at the design, operation and maintenance stages of EDF's PWRs aims at ensuring a coherent management of individual and collective exposures. The objective of such a policy is, as recommended by ICRP 60 , to keep exposures (number of individuals exposed and levels of exposure) as low as reasonably achievable, with an equitable distribution taking into consideration economical and social aspects. This leads EDF to adopt, among other tools, a system of reference monetary values for the unit of collective dose averted. After having recalled the ethical issue associated with such an approach as well as the international consensus on the dose-risk relationship for low doses, the model adopted, integrating risk aversion considerations, is presented. Its main characteristic is a rapid increase of the monetary values with the level of annual individual doses coming close to the regulatory limits. This model will be used as a decisionaiding tool for the evaluation and comparison of altemative protection actions within the optimisation process of workers'exposure.

* EDF SEPTEN, 12-14, av. Dutrievoz, 69628 Villeurbanne Cedex.

** EDF DSRE, 6, rue Ampère, BP 114, 93203 Cedex 1.

*** Centre d'étude sur l'évaluation de la protection dans le domaine nucléaire (CEPN), BP 48, 92263 Fontenay-aux-Roses Cedex.

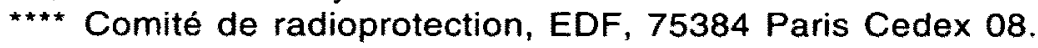




\section{Introduction}

EDF a construit son parc électronucléaire en prenant des dispositions visant à limiter les sources de rayonnement dès la conception, dans la limite de ce que permettaient le rythme de réalisation du parc et la volonté de standardisation. Cependant, cette démarche a été effectuée sans souci particulier d'optimisation de la radioprotection. Les excellents résultats enregistrés pendant 10 années (dose collective moyenne annuelle inférieure à 2 homme-sievert; doses sur le palier $1300 \mathrm{MW}$, le plus récent, inférieures à celles observées sur le palier $900 \mathrm{MW}$ ) témoignent de la réalité des dispositions prises et de leur efficacité. La dégradation progressive de ces mêmes résultats depuis 1988 (2,58 homme.Sv en 1991) en souligne les limites.

II apparaît clairement que la maîtrise de la dose collective, tant en valeur absolue qu'en termes de répartition sur les divers acteurs, passe par une approche rationnelle de la maîtrise de chacun des facteurs qui interviennent dans la constitution de la dose collective : débit d'équivalent de dose, temps d'exposition, nombre d'opérateurs. Une telle approche, communément appelée optimisation de la radioprotection ou "ALARA" (as low as reasonably achievable, c'est-à-dire aussi bas que raisonnablement possible) permet d'évaluer l'efficacité des actions de radioprotection en tenant compte du contexte économique et social. La communauté internationale dans le domaine du nucléaire civil s'accorde pour reconnaître qu'il n'est pas nécessaire d'avoir la preuve des effets biologiques des rayonnements ionisants aux faibles doses pour s'engager résolument dans une approche "ALARA" : il suffit d'être convaincu de l'intérêt intrinsèque de cette méthode de gestion (définition d'objectifs/mesure des performances/analyse des écarts et retour d'expérience).

De nombreux facteurs ont donc incité EDF à adopter sans réserve cette approche :

- la volonté de maîtriser l'exposition lors des opérations d'exploitation et de maintenance du parc comme cela a été réalisé lors de l'opération de remplacement des générateurs de vapeur de la centrale de Dampierre 1 , et d'éviter la dérive des doses collectives avec le vieillissement des installations ;

- les nouvelles recommandations de la Commission internationale de protection radiologique (CIPR) (publication $n^{\circ} 60$ ) [10] qui doivent conduire à la fois à limiter le nombre de travailleurs exposés, leur exposition individuelle sur 5 ans à une valeur au plus égale à $100 \mathrm{mSv}$, et à obtenir une répartition du risque plus équitable ;

- la conception de nouveaux réacteurs qui doit permettre d'intégrer dès cette phase les considérations dosimétriques d'exploitation et de maintenance, en tenant compte du retour d'expérience international qui montre clairement les progrès qui peuvent être réalisés à ce niveau ;

- le souci d'affecter les ressources là où elles sont les plus efficaces. 
Pour concrétiser ces objectifs, il est indispensable de disposer d'un ensemble d'outils adaptés pour mettre en œuvre l'approche ALARA. La fixation d'un système de valeur monétaire de référence de l'unité de dose collective est un élément central de tout programme ALARA. L'article présente la méthodologie retenue par EDF pour fonder un tel système, ainsi que les valeurs adoptées. II est le fruit des travaux réalisés par le CEPN en collaboration avec EDF [11-12].

\section{Remarques préliminaires}

Le débat scientifique actuel ne permet pas plus de démontrer qu'il existe un risque dû aux faibles expositions que de démontrer le contraire. L'adoption d'une relation linéaire dose-effet avec les coefficients de risque de la CIPR 60 correspond donc à une attitude prudente des experts internationaux, relayée par les décideurs politiques. Cette attitude prudente est courante en matière de gestion des risques collectifs.

Fixer une valeur monétaire à l'unité de dose collective revient à donner une valeur à la perte d'espérance de vie et même à la détérioration de la qualité de la vie, en raison même de l'adoption de la relation dose-effet. Cela ne va pas sans soulever des problèmes d'éthique car on peut, à juste titre, considérer que la vie d'un individu n'a pas de prix. Cependant, dans le domaine de la prévention, pour éviter des risques statistiques, des choix doivent être faits car les ressources ne sont pas illimitées et toute décision en matière de prévention se traduit par une "valeur" implicite de la vie humaine.

Cette logique a déjà été adoptée explicitement dans de nombreux domaines comme celui des infrastructures routières par exemple [5, 13]. Se doter d'un système de valeurs de référence de l'unité de dose collective évitée revient donc à appliquer au domaine de la radioprotection une rationalité tout à fait admise et pratiquée dans nos sociétés.

\section{Définitions}

Pour éviter de nombreuses confusions, il paraît utile de préciser quelques définitions.

\subsection{Les coûts de la protection}

La mise en cuvre d'actions de protection induit des coûts d'investissement et éventuellement d'exploitation-maintenance qui traduisent l'ampleur et la complexité des systèmes envisagés : protections biologiques, outillages spécialisés, modifications d'équipements... Les coûts de protection sont généralement assez facilement appréhendables, bien que, dans certaines circonstances, il soit difficile de faire la part dans les dépenses de ce qui relève de la protection et d'autres fonctions comme la production ou la sûreté. 


\subsection{Surcoût nucléaire}

La nécessité de réaliser des opérations en milieu hostile conduit à prendre certaines précautions concrétisées en milieu nucléaire par l'existence d'une zone contrôlée. Cela implique des coûts supplémentaires par rapport à un travail en milieu normal ou sans risque. Ce surcoût nucléaire est constitué par des coûts fixes correspondant à l'infrastructure de radioprotection, et des coûts variables tels que la formation des intervenants, l'obligation de porter des tenues vestimentaires adaptées (sources de déchets dont la gestion est elle-même coûteuse, et genératrices d'inconfort et de perte de productivité). On retiendra que ces coûts, qui incluent les coûts de la protection, ne sont pas toujours aisés à déterminer.

\subsection{Coûts de l'unité de dose évitée}

A toute action de radioprotection peuvent être associés son efficacité et son coût réel. Le coût de l'unité de dose évitée n'est autre que le ratio coût réel / dose évitée ( $F /$ homme-sievert). Ce coût est donc spécifique à une action de radioprotection. Toute action visant à réduire la dose aura donc un impact direct en termes de dépenses de protection (le coût de protection) et un impact potentiel indirect sur le surcoût nucléaire. L'expérience montre, en effet, que dans de nombreux cas une action de protection pourra conduire à des réductions des coûts qu'il conviendra de ne pas oublier dans le bilan global de l'action. Ainsi, par exemple, le remplacement de l'homme par un robot pour effectuer une tâche, coûteux en termes d'investissement, sera aussi à l'origine de gains de productivité, de réduction du nombre de tenues vestimentaires spéciales et des déchets associés.

\subsection{Valeurs de référence}

Le système de valeurs de référence de l'unité de dose est, contrairement au coût de l'unité de dose évitée, indépendant de l'action de radioprotection envisagée. II s'agit d'un jeu de valeurs de référence jugées raisonnables pour éviter une unité de dose collective, faisant l'objet d'une décision politique au sein de l'organisme qui l'a choisi. C'est à elles que seront comparés les coûts de l'unité de dose évitée associés aux diverses options de radioprotection.

\section{Les principes du système de référence}

\subsection{Prise en compte de la CIPR 60}

La nouvelle formulation du principe d'optimisation ALARA dans la publication $n^{\circ} 60$ de la CIPR précise clairement que :

"l'importance des doses individuelles, le nombre de personnes exposées doivent être maintenus aussi bas qu'il est raisonnablement possible, compte tenu des facteurs économiques et sociaux. Cette procédure (d'optimisation) doit être mise en œuvre avec des restrictions sur les doses individuelles afin de limiter l'inéquité susceptible de résulter des situations" [10]. 
Ainsi, outre l'objectif de réduction optimale de la dose collective énoncé dès 1977 (CIPR 26) [7], la CIPR 60 recommande de réduire le nombre de personnes exposées, et de réduire la dispersion des doses individuelles, en tirant leur valeur vers le bas (elle doivent être en moyenne sur 5 ans inférieures à $20 \mathrm{mSv} / \mathrm{an}$ ), le maximum réglementaire restant à $50 \mathrm{mSv} / \mathrm{an}$. La CIPR introduit donc explicitement la notion d'équité dans les fondements de l'optimisation. Cette idée est loin d'être une nouveauté puisque l'on peut en trouver une première mention dès la publication 22 (1973) [6] de la CIPR et que dans les publications 37 (1983) [8] et 42 (1984) [9] une formulation a été proposée pour prendre en compte les considérations associées à la distribution des doses individuelles. C'est sur ces bases que le National radiological protection board (NRPB) a élaboré le premier système de valorisation monétaire de l'unité de dose avec des valeurs croissantes en fonction du niveau des doses individuelles [2] et que le CEPN a proposé, dès 1988, trois valeurs de l'unité de dose correspondant à des plages successives de doses individuelles pour les travailleurs [12].

Sur le plan pratique, il est bien établi que, dans plusieurs domaines, les distributions de doses individuelles sont assez larges avec même, dans quelques cas, une part non négligeable d'opérateurs recevant des doses proches des limites réglementaires. C'est, en particulier, le cas pour le personnel des entreprises extérieures qui assurent l'essentiel des travaux de maintenance dans les réacteurs.

Le système de référence qui a été adopté respecte l'ensemble de ces principes et, bien entendu, tient compte de la modification des coefficients de risque de la CIPR. II correspond, par ailleurs, aux situations d'exposition des travailleurs lors du fonctionnement normal des installations ; il ne concerne pas les situations accidentelles ni l'exposition du public tant à court qu'à moyen ou long terme.

\subsection{Le modèle retenu}

Il correspond à la volonté de prise en compte du principe d'équité via la notion d'aversion pour la dispersion des risques. Si l'on ne prenait pas en compte la notion d'équité il serait possible de se contenter d'une valeur unique de référence pour l'unité de dose évitée indépendante du niveau d'exposition des individus.

En pratique, dans le domaine de la gestion des risques, ĺa notion d'équité est généralement considérée comme équivalente à celle de l'aversion pour la dispersion des risques. Un tel concept, très fréquemment utilisé dans le domaine de la gestion des risques, traduit le fait que I'on est prêt à payer davantage pour éviter une unité d'un risque donné lorsque le niveau de risque augmente. Dans notre domaine, compte tenu de l'hypothèse de croissance linéaire du risque avec l'exposition, cela revient à dire que l'on est prêt à payer davantage pour éviter une unité de dose lorsque les doses individuelles augmentent. 
II s'agit d'une approche collective, dans laquelle un décideur doit "gérer" un ensemble de risques individuels. Cette approche a son corollaire au niveau des décisions individuelles avec la notion d'aversion pour le risque qui correspond directement à l'aversion croissante qu'un individu éprouve face à des niveaux de risque de plus en plus élevés.

L'hypothèse est généralement admise qu'en dessous d'un certain niveau de risque individuel, le phénomène d'aversion ne joue pas. Dans le cas de l'exposition aux rayonnements ionisants cela revient à dire qu'en dessous d'un certain niveau d'exposition individuelle, la valeur monétaire de l'unité de dose est une valeur constante. Au-dessus de ce niveau, l'aversion à la dispersion des risques se traduit par un coefficient d'aversion. Le modèle retenu pour déterminer les valeurs de référence de l'unité de dose intègre le degré d'aversion de la façon suivante :

où

$$
\alpha_{i \text { ref }}=\alpha_{\text {base }}\left(D_{i} / D_{0}\right)^{a}
$$

$\alpha_{\text {base }}=$ valeur monétaire de base de l'unité de dose collective quand le phénomène d'aversion à la dispersion des risques ne joue pas,

$D_{0} \quad=$ bome supérieure de la plage de dose individuelle annuelle pour laquelle $\alpha_{\text {base }}$ est applicable,

$D_{i} \quad=$ niveau de dose individuelle annuelle,

a = coefficient représentant l'aversion pour la dispersion des risques,

$\alpha_{i \text { ref }}=$ valeur monétaire de référence de l'unité de dose collective pour le niveau d'exposition individuelle $i$.

Pour obtenir un système de valeurs de référence de l'unité de dose collective, il est donc nécessaire de fixer les valeurs des différents paramètres : $\alpha_{\text {base }} ; D_{0}$ et $\alpha$.

\subsection{Les valeurs retenues pour les paramètres}

Valeur de $\alpha_{\text {base }}$

La valeur d' $\alpha_{\text {base }}$ pour la plage dosimétrique où l'aversion à la dispersion des risques ne joue pas $(a=0)$ est déterminée à partir de considérations sur la perte d'espérance de vie qui est statistiquement associée à une unité de dose collective, sous l'hypothèse de la relation linéaire doseeffet, ainsi qu'aux dépenses de santé engendrées par le traitement des cancers. Plusieurs méthodes ont été développées dans la théorie économique pour évaluer les coûts correspondants [11-12]. La méthode retenue ici est celle qui rattache ces coûts aux données de la vie économique, en particulier à l'évaluation de la contribution des individus à l'économie nationale par l'intermédiaire du produit national brut (PNB) par habitant. Cette méthode est une approche qui, pour rudimentaire qu'elle soit, a au moins deux mérites : elle est retenue par l'Agence internationale de l'énergie atomique (AIEA), et elle n'introduit pas de distinction entre les diverses catégories d'individus. 
Sur ces bases avec les coefficients de risque de la CIPR 60 (traduits par une perte d'espérance de vie de 0,88 année pour 1 homme.Sv dans le cas de l'exposition professionnelle) et un PNB/habitant en France de $108 \mathrm{kF}$ (1989), on obtient une valeur d' $\alpha_{\text {base }}$ arrondie à $100 \mathrm{kF} / \mathrm{homme} \cdot \mathrm{Sv}$ (1992) [11].

\section{Valeur de $\mathrm{D}_{0}$}

II paraît judicieux de retenir la limite d'exposition pour le public comme valeur de $D_{0}$ pour les expositions professionnelles : l'avantage est de faire référence à une situation jugée "acceptable" et, qui plus est, de se situer au niveau de l'irradiation naturelle. Par conséquent, la valeur de 1 $\mathrm{mSv} / \mathrm{an}$ a été adoptée pour $D_{0}$.

\section{Valeur de a}

L'examen des valeurs de a employées dans des études économiques similaires [16] conduit à retenir une plage de variation possible des valeurs de a comprise entre 1,2 et 1,5 pour le domaine de l'exposition professionnelle aux rayonnements ionisants. A titre tout à fait indicatif, une valeur de 1,8 correspondrait aux risques consécutifs aux catastrophes naturelles pour les individus du public.

Les valeurs de référence possibles correspondant à $\alpha_{\text {base }}=100 \mathrm{kF}$, $D_{0}=1 \mathrm{mSv} / \mathrm{an}$ et a compris entre 1,2 et 1,5 , sont indiquées dans le tableau 1 et représentées graphiquement sur la figure 1.

TABLEAU I

Valeurs de référence de l'homme-sievert en fonction du niveau de dose individuel annuel et de la valeur du coefficient d'aversion pour la dispersion des risques Man-sievert reference values vs annual individual dose levels and risk dispersion aversion coefficient values

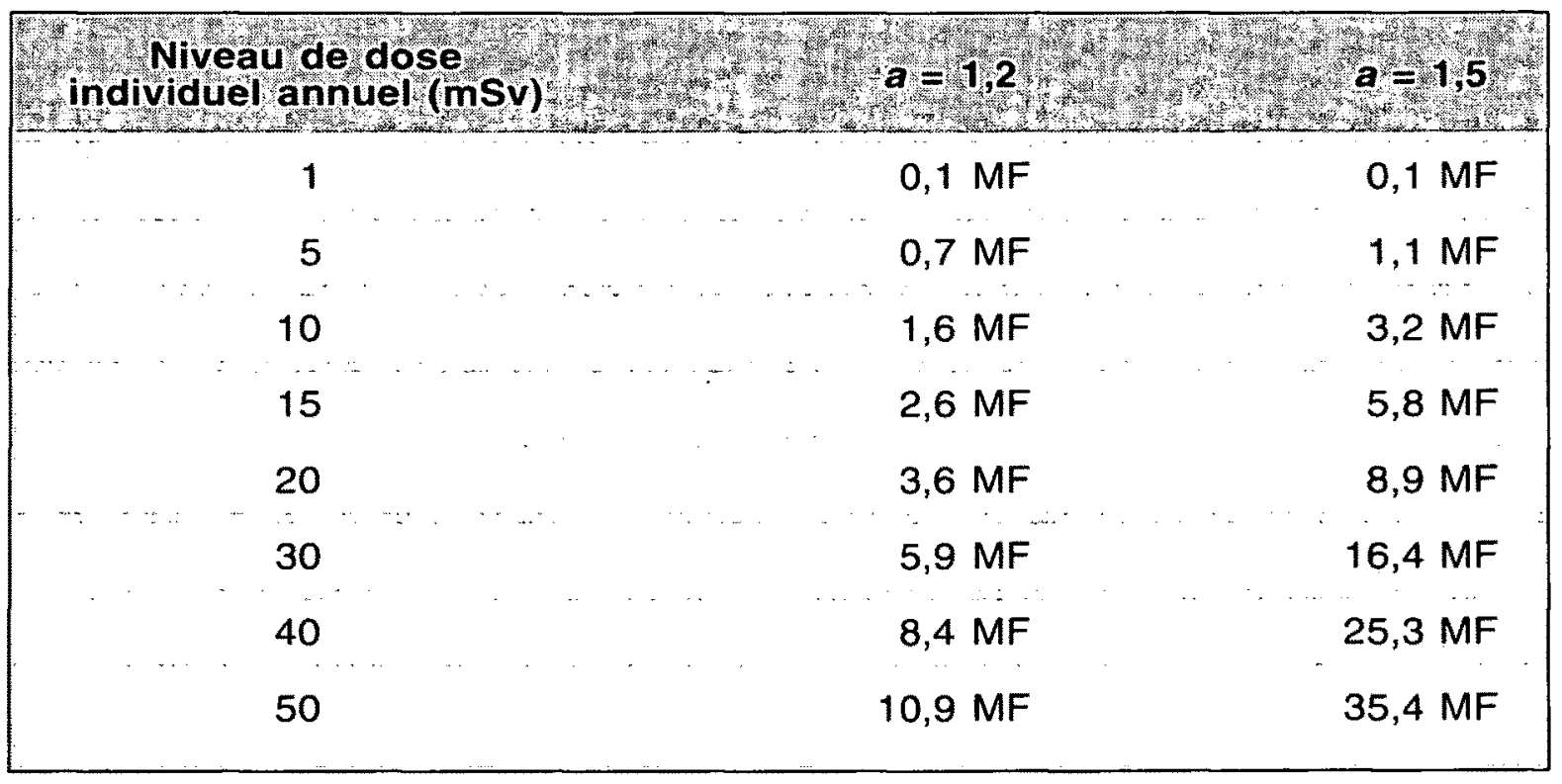




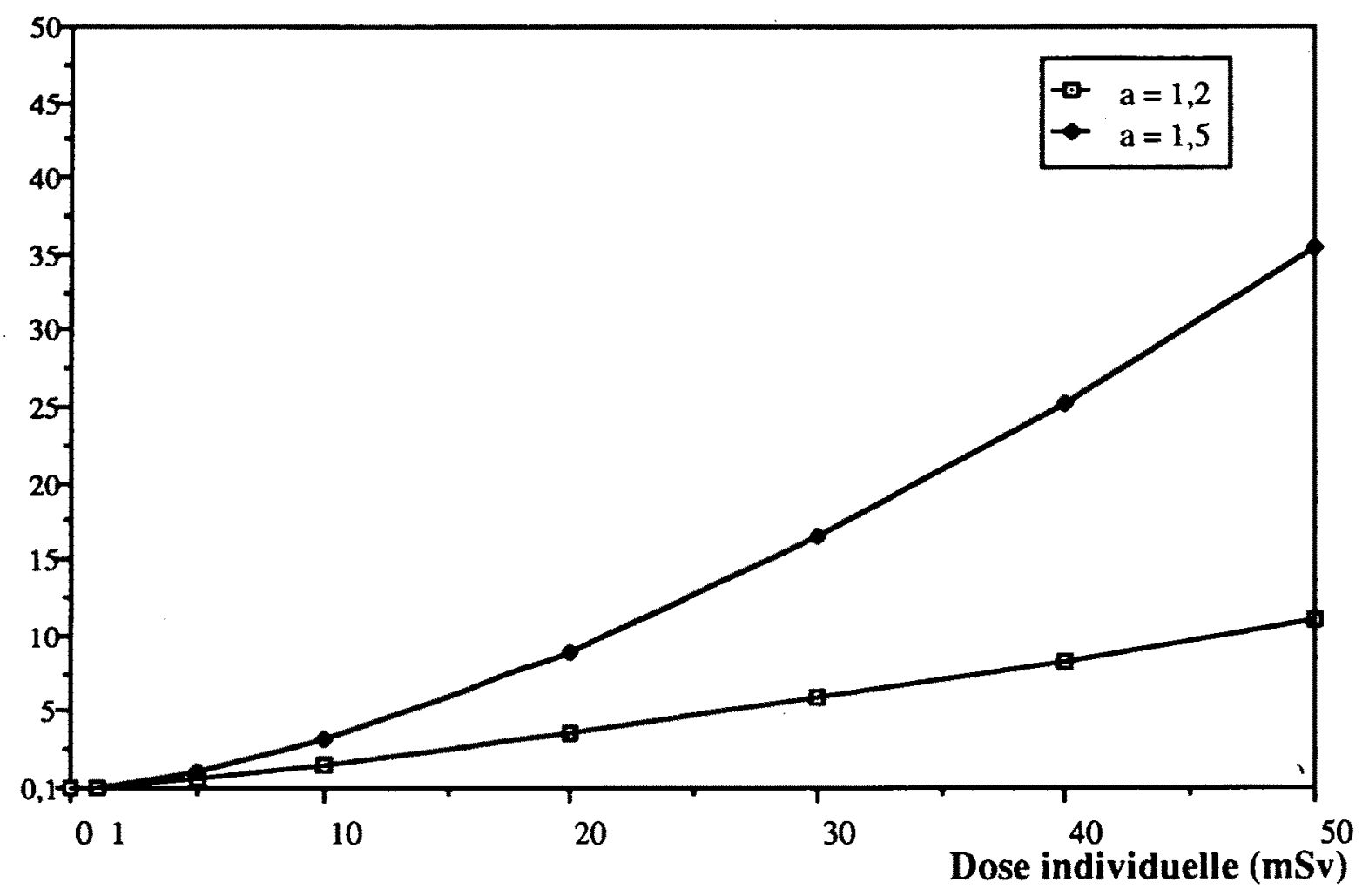

Fig. 1. - Valeur de référence de l'homme.sievert en fonction du niveau de dose individuel annuel.

Range of man-sievert reference values.

Désireux d'effectuer un effort, à la fois significatif et raisonnable, en vue de diminuer l'exposition collective professionnelle, le nombre d'opérateurs exposés et la dispersion des expositions individuelles, EDF a retenu, pour une période de l'ordre de deux ans, une valeur moyenne de a égale à 1,35. Les études d'optimisation réalisées au sein de l'entreprise devront donc retenir cette valeur et effectuer une analyse de sensibilité allant de $a=1,2$ à $a=1,5$ pour tester la robustesse des résultats.

Le système des valeurs de référence devant être simple, il est apparu souhaitable de remplacer pour la majorité des applications pratiques la valorisation continue par une valorisation en "marches d'escalier". Les plages dosimétriques retenues correspondent aux valeurs suivantes : $5 \mathrm{mSv} / a n$ (ancienne limite public), $15 \mathrm{mSv} / \mathrm{an}$ ("clignotant" destiné à rappeler la proximité de la valeur moyenne annuelle de $20 \mathrm{mSv}$ ), 30 et $50 \mathrm{mSv} / a n$ pour la dernière plage (valeurs maximales trimestrielles et annuelles). Avec une valeur de a de 1,35 et les plages ci-dessus on obtient (valeurs arrondies) : 
TABLEAU $\|$

Valeurs de l'homme sievert retenues en fonction des plages de doses annuelles Man-sievert values considered vs annual dose ranges

\begin{tabular}{|l|}
$\alpha_{0-1} \mathrm{mSv} / \mathrm{an}=0,1 \mathrm{MF} / \mathrm{homme} \cdot \mathrm{Sv}$ \\
$\alpha_{1-5} \mathrm{mSv} / \mathrm{an}=0,5 \mathrm{MF} / \mathrm{homme} \cdot \mathrm{Sv}$ \\
$\alpha_{5-15} \mathrm{mSv} / \mathrm{an}=2,3 \mathrm{MF} / \mathrm{homme} \cdot \mathrm{Sv}$ \\
$\alpha_{15-30} \mathrm{mSv} / \mathrm{an}=6,7 \mathrm{MF} / \mathrm{homme} \cdot \mathrm{Sv}$ \\
$\alpha_{30-50} \mathrm{mSv} / \mathrm{an}=15,0 \mathrm{MF} / \mathrm{homme} \cdot \mathrm{Sv}$
\end{tabular}

La valeur moyenne de la fonction a sur l'intervalle 0-50 est alors proche de $10 \mathrm{MF} / \mathrm{homme} \cdot \mathrm{Sv}$ (soit $100000 \mathrm{~F} / \mathrm{homme}-\mathrm{rem}$, ou encore 20000 \$/homme-rem).

Si l'on admet qu'un haut degré de protection a été obtenu dans le domaine du nucléaire par rapport à d'autres industries, mais que certains progrès restent à faire, le système ainsi défini permettra que les ressources soient systématiquement allouées en priorité aux situations les plus pénalisantes sur le plan dosimétrique. II doit alors permettre de faire disparaître progressivement les expositions annuelles voisines de la limite réglementaire et faciliter le respect des $100 \mathrm{mSv}$ sur 5 ans. Les modalités d'application de la méthode font l'objet d'une note interne EDF.

\section{Comparaison internationale}

L'objectif ici n'est pas de dresser un bilan exhaustif de la situation sur le plan international mais de discuter les valeurs adoptées par EDF à la lumière de celles couramment utilisées, ou qui viennent d'être réévaluées, dans trois pays assez différents sur le plan du contexte socio-économique. Le tableau III présente donc un certain nombre de valeurs de l'homme.Sv utilisées dans d'autres pays.

Au vu de ce tableau, il apparaît clairement que les systèmes utilisés sont, ou vont, être revus suite à la CIPR 60. Par ailleurs, il faut noter que les valeurs suédoises sont des valeurs planchers contractuelles. Audessus de ces valeurs les décisions sont discutées, en particulier au vu de la distribution des doses individuelles. Enfin, en Grande-Bretagne, les valeurs recommandées par le NRPB apparaissent largement dépassées par l'industrie, comme en témoigne la valeur utilisée par British nuclear fuel. EDF se situe donc dans la plage supérieure de la fourchette des valeurs utilisées dans l'industrie nucléaire. 
TABLEAU III

Valeurs de l'homme-sievert utilisées ou proposées à l'étranger Man-sievert values used or proposed in foreign countries

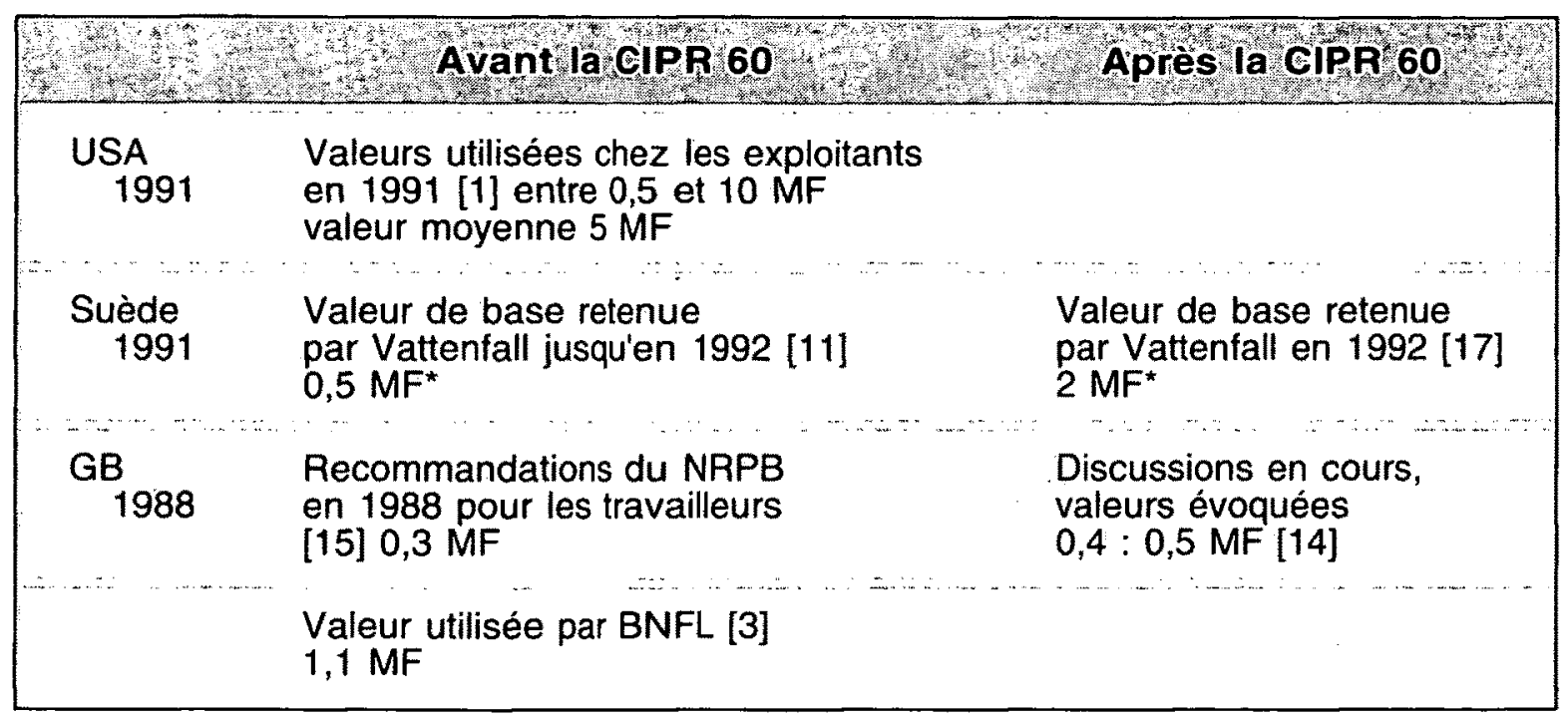

\section{Conclusion}

La méthodologie qui amène au système de valeurs de référence de l'unité de dose collective adoptée par EDF permet objectivement de respecter l'esprit des recommandations de la CIPR. Le choix de la valeur du coefficient d'aversion à la dispersion des risques lui-même permet de moduler le niveau de protection afin d'obtenir une cohérence avec la consistance du problème dosimétrique associé au parc REP d'une part, et aux possibilités de financement d'autre part.

Le système, qui fait l'objet d'un consensus à EDF, est mis en application dans l'entreprise et est commun aux domaines de la conception, de l'exploitation et de la maintenance des réacteurs. II garde son caractère d'outil d'aide dans des processus de décision inévitablement complexes.

II apparaît hautement souhaitable que ce système reste de la responsabilité de l'entreprise, l'Etat conservant quant à lui ses rôles d'incitation et de contrôle en matière de protection des individus. Cependant, cette méthodologie nécessitera l'établissement d'un échange entre l'ensemble des exploitants et concepteurs, le corps médical et l'administration afin de permettre un dialogue fondé sur un vocabulaire commun.

On aura soin de conserver une flexibilité importante au système en fixant une durée de vie adaptée à un jeu de valeurs de référence. Ceci est justifié par le fait que la situation dosimétrique est et restera évolutive et que les valeurs de références n'ont manifestement pas de caractère absolu. 
Enfin, on peut raisonnablement s'attendre à ce qu'une gestion de la radioprotection rationnelle, et donc nécessairement plus transparente, soit une source réelle d'économies avec un niveau de protection pourtant augmenté. Les valeurs de référence très élevées n'apparaîtraient alors que comme des incitations à faire en sorte qu'on n'ait pas à les employer.

\section{RÉFÉRENCES}

[1] BAUM J.W. - Valuation of dose avoided at U.S. nuclear power plants. Nucl. Plant J., 1991, 9 (2), 40-47.

[2] CLARK M.J., FLEISHMAN A.B. - The cost of collective dose equivalent CEA/NRPB/UKAEA Joint technical meeting on cost benefit analysis, Chilton, 29-30 March 1979, Chilton : NRPB, 1979.

[3] COATES R. - ALARA in major refurbishment at BNFL. CEC ALARA training course, Saclay, INSTN, novembre 1990.

[4] EGNER K. - ALARA in Sweden, exposé au Cours de formation CCE, optimisation de la radioprotection dans la conception et l'exploitation des installations nucléaires et industrielles, Saclay (France), INSTN, 4 au 8 novembre 1991.

[5] FRANCE. Ministère de l'équipement, SAEI. Etude pilote RCB sécurité routière. 1967-1970.

[6] INTERNATIONAL COMMISSION OF RADIOLOGICAL PROTECTION (ICRP) Implication of Commission recommendations that doses be kept as low as readily achievable. (ICRP Publication 22). Oxford: Pergamon press, 1973.

[7] INTERNATIONAL COMMISSION OF RADIOLOGICAL PROTECTION (ICRP) Recommandations... (ICRP Publication 26). Oxford: Pergamon Press, 1977.

[8] INTERNATIONAL COMMISSION OF RADIOLOGICAL PROTECTION (ICRP) Cost-benefit analysis in the optimization of radiation protection. (ICRP Publication 37). Oxford : Pergamon press, 1983.

[9] INTERNATIONAL COMMISSION OF RADIOLOGICAL PROTECTION (ICRP) - A compilation of the major concepts and quantities in use by ICRP. (ICRP Publication 42). Oxford : Pergamon press, 1985.

[10] INTERNATIONAL COMMISSION OF RADIOLOGICAL PROTECTION (ICRP) Recommendations... (ICRP Publication 60) - Oxford : Pergamon press, 1991.

[11] LEFAURE C., LOCHARD J., SCHNEIDER T., SCHIEBER C. - Proposition pour un système de valeurs de référence de l'unité de dose collective. CEPN-R-193, 1992.

[12] LOMBARD J. - Proposition de valeurs de référence de I'homme - rem pour l'exposition professionnelle. CEPN-R-136, 1988.

[13] MATTSON B. - Priset for var Sakerhet, Rapport till RRV, Rapport 551-92-03, Stockholm, 1992.

[14] NATIONAL RADIOLOGICAL PROTECTION BOARD (NRPB) - communication personnelle.

[15] ROBB J.D., WRIXON A.D. - Revised estimates of the monetary value of collective dose. NRPB-M157, 1988.

[16] SCHIEBER C., SCHNEIDER T., PAGES P. - L'aversion au risque dans le cadre de l'optimisation de la radioprotection : les enseignements de la théorie du risque et de l'assurance. CEPN-NTE/92/003.

[17] VATTENFALL - communication personnelle. 